\title{
Fluorescent Probe for Simultaneous Detection of Human Serum Albumin and Sulfur Dioxide: A Theoretical Analysis
}

\author{
ang jia ${ }^{1}$, he huang ${ }^{2}$, zhong-fu zuo ${ }^{2}$, xuezheng liu², and Yongjin Peng ${ }^{2}$ \\ ${ }^{1}$ First Affiliated Hospital of Jinzhou Medical University \\ ${ }^{2}$ Jinzhou Medical University
}

September 29, 2021

\begin{abstract}
A small molecule probe for simultaneous detection of HSA and SO2 via their distinct fluorescent signals was designed recently. This effective tool provided a significant boost in understand underlying mechanism of synergistic action between SO2 and HSA in disease. The structure and fluorescent character of this probe molecule were studied under density functional theory in this work. The different stable conformations of probe C23 were found through theoretical method which explained the no experimental fluorescent character of the probe itself. The electron excitation analysis indicated the charge transfer process in the restricted C23 (binding to the hydrophobic cavity of HSA) and CS (C23 reaction with SO2) when the molecules were under optical excitation. The theoretical results could be helpful for understanding the electronical properties in the probe and providing the insights for designing new probe molecules.
\end{abstract}

\section{Introduction}

Studies have shown that $\mathrm{SO}_{2}$-induced HSA free radicals could cause tissue damage during allergic reactions, eosinophilic inflammation, and lung disease. ${ }^{1-6}$ An effective tool of simultaneous detection of $\mathrm{HSA}$ and $\mathrm{SO}_{2}$ is important for understanding the underlying mechanism of synergistic action between $\mathrm{SO}_{2}$ and $\mathrm{HSA}$ in disease. Recently Liang et al designed a fluorescent small molecule probe for simultaneous detection of HSA and $\mathrm{SO}_{2}$ via their distinct fluorescent signals. ${ }^{7}$ The design concept was as showed in Scheme 1.

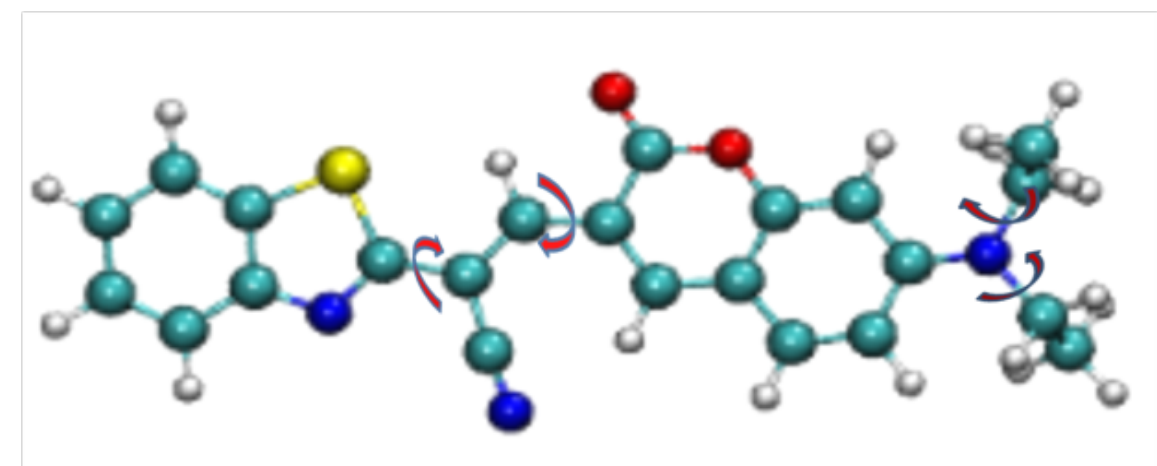

Scheme 1 probe C23

The probe itself does not fluoresce due to intramolecular rotation. In the presence of HSA, the probe molecules specifically bind to the hydrophobic cavity of HSA, causing the probe molecules to be unable to 
rotate, resulting in strong red fluorescence. When the probe coexists with $\mathrm{SO}_{2}$ alone, a specific Michael addition reaction occurs (as shown in Scheme 2), which reduces the $\pi$-conjugated structure of the probe and produces obvious blue fluorescence.

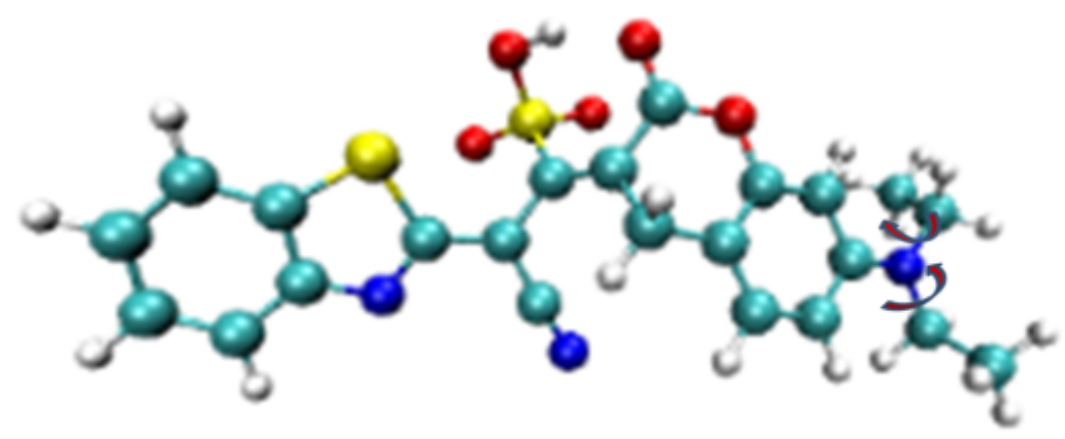

Scheme 2 CS-Michael addition reaction of probe $\mathrm{C} 23$ and $\mathrm{SO}_{2}\left(\mathrm{HSO}_{3}{ }^{-1}\right.$ in the solution)

When the probe is combined with HSA, the Michael addition reaction between the probe and $\mathrm{SO}_{2}$ is rapidly catalyzed by HSA, and this catalytic effect of HSA greatly improves the detection sensitivity of $\mathrm{SO}_{2}$. Studies have shown that the amino group of Lys199 in HSA contributes most of the catalytic activity. ${ }^{7}$ The results of liang et al showed good performance of this probe molecule for the simultaneous determination of HSA and $\mathrm{SO}_{2}$ molecules in urine and cells. And this probe was expected to be used in the diagnosis of liver cancer, drug evaluation, and the study of physiological and pathological functions of $\mathrm{HSA}$ and $\mathrm{SO}_{2}$. In this work, the structure and fluorescent character of this probe molecule were studied under density functional theory. The rotation of the unsaturated C-C bonds in the probe molecule led to several stable structures coexisted. The transfer between different stable structures provided the non-radiative energy loss pathway when the probe molecule recovered from the excited state to the ground state which led to no fluorescence in this process. The calculated results indicated the red fluorescence would be generated if the rotation of the unsaturated $\mathrm{C}-\mathrm{C}$ bonds in the probe molecule was prohibited such as the probe molecule was stabilized in the hydrophobic cavity of HSA. The calculation also indicated that the Michael reaction between the probe molecule and $\mathrm{SO}_{2}$ would be another effective way for the stabilization of the probe molecule and generating blue fluorescence which made this probe molecule is able of simultaneous determination of HSA and $\mathrm{SO}_{2}$ molecules. All the figures were rendered by means of VMD 1.9.3 software ${ }^{8}$ and the analyses were finished by using the Multiwfn 3.7 code $^{9}$.

\section{Method}

The processes of the calculation were as follows:

(1) Generate initial conformations of probes with Confab; ${ }^{10}$

(2) Use Crest to call xtb to do batch structural optimization under the GFN2-xTB method, ${ }^{11}$ and then call isostat in Molclus ${ }^{12}$ program to screen out several conformations with the lowest energy for next step;

(3) Use Molclus to call $\mathrm{ORCA}^{13}$ to perform optimization and vibrational frequency analysis on the conformations generated from step (2) under PBE0/def2-TZVP, ${ }^{14},{ }^{15}$ and then single point energy and TDDFT calculation under wB2GP-PLYP/def2-TZVP so as to obtain the free energy with high precision. ${ }^{16,17}$

\section{Results and Discussion}

The four most stable conformations of probe C23 were found as shown in Figure1. The difference among the four conformations were clearly depicted in the front and side view of the molecular structure of the probes 
due to the rotational bonds. The transfer among the different conformations provided the non-radiation energy loss when the probe $\mathrm{C} 23$ recovered back to the ground state from excited states, led to no fluorescence generation with the probe $\mathrm{C} 23$ itself. When the probe was incorporated into a hydrophobic cavity of HSA, the bonding interaction between the probe and HSA resulted in the inhibition of non-irradiative energy loss pathways and an increase in fluorescence within the red channel.
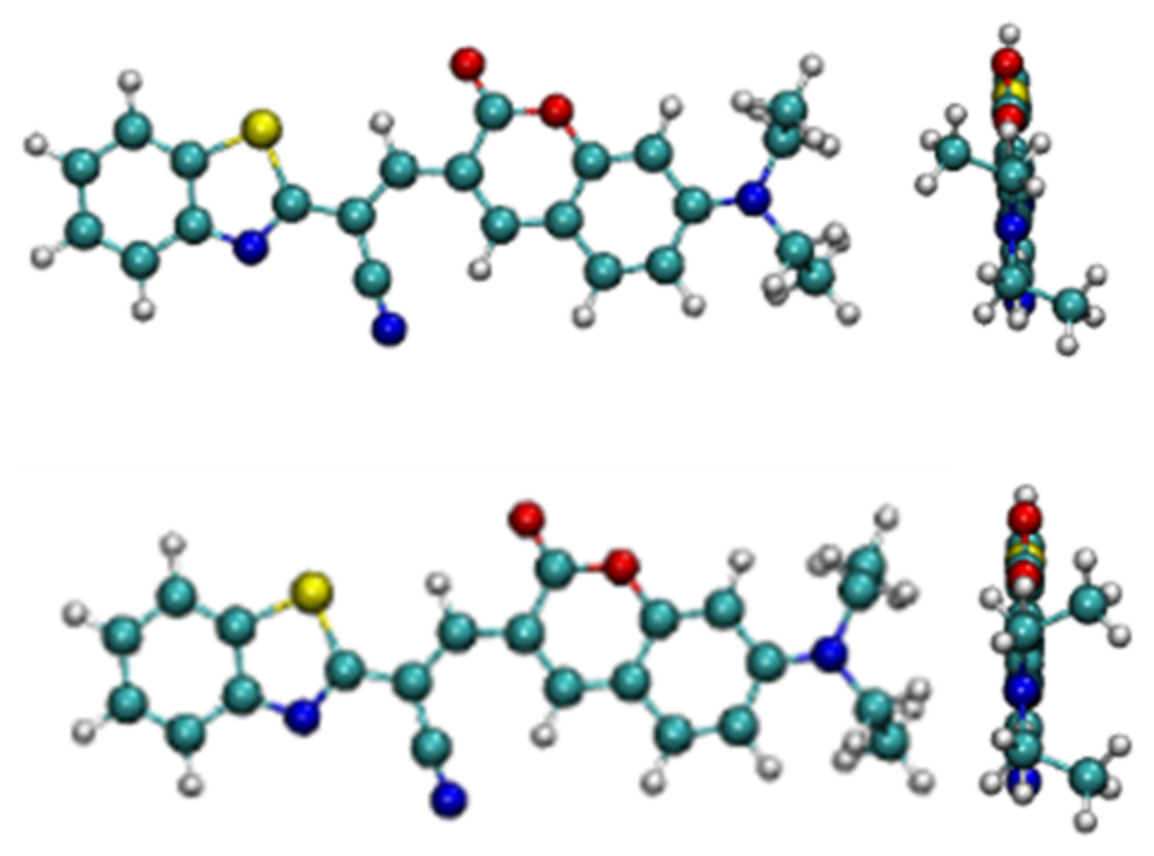

C23-1 C23-2

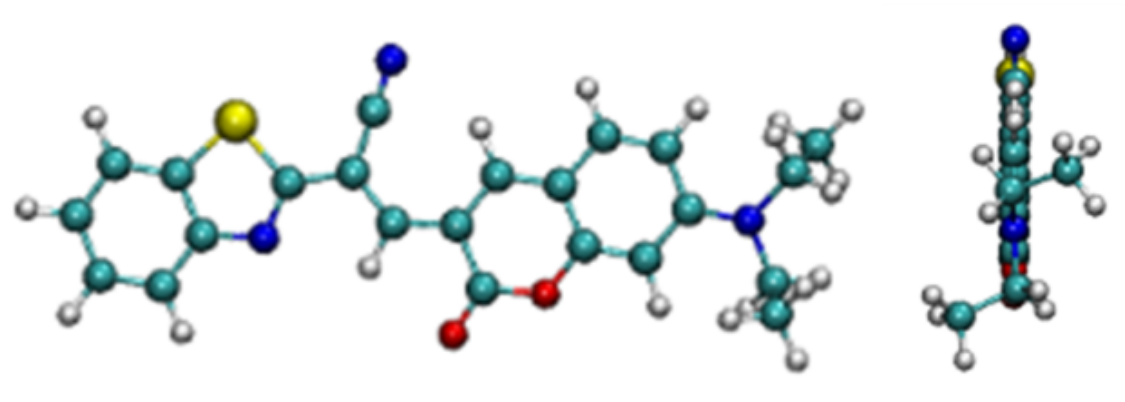

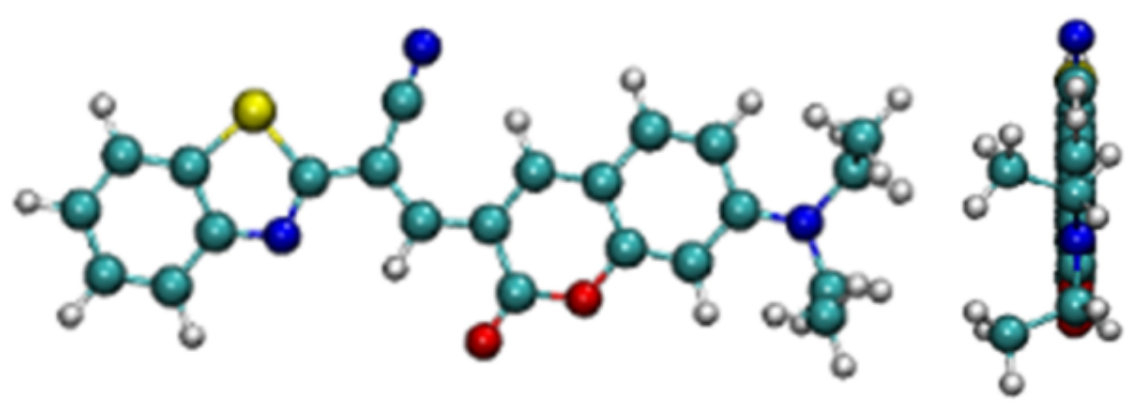




\section{C23-3 C23-4}

Figure1 Four most stable conformations of probe $\mathrm{C} 23$

To illustrate the reaction sites within the probe molecule, the electrostatic potential of C23-1 was analyzed using Multiwfn 3.7 from the wave function generated under wB2GP-PLYP/def2-TZVP combination from ORCA program. The electrostatic potential of C23-1 was as shown in Figure2. Several local maximum and minimum values of electrostatic potential except those within the $\mathrm{N}_{-} \mathrm{CH}_{2}-\mathrm{CH}_{3}$ groups were depicted in the Figure2 which showed the potential reaction sites with the HSA. The results were agreed with the previous work. $^{7}$
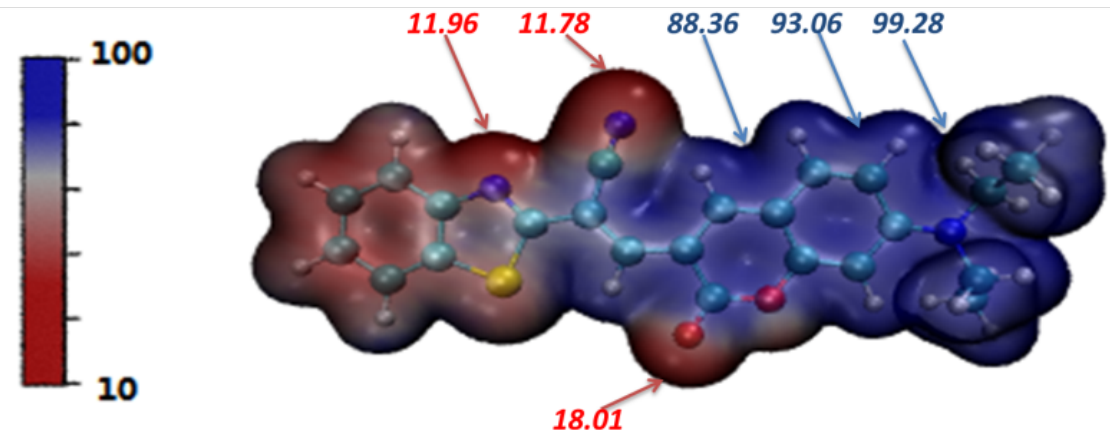

Figure2 Electrostatic potential of C23-1

The electron excitation process within the probe C23 was analyzed under TDDFT method within the ORCA program. The structure difference between the ground state $\mathrm{S}_{0}$ and lowest excited state $\mathrm{S}_{1}$ were small for all four conformations. The electron density difference between the $\mathrm{S}_{0}$ and $\mathrm{S}_{1}$ as shown in Figure3a and Figure3b for $\mathrm{C} 23-1$ and $\mathrm{C} 23-4$ respectively could show the electron transfer in the excitation process. The electron density difference between the $\mathrm{S}_{0}$ and $\mathrm{S}_{1}$ in C23-2 and C23-3 were similar to C23-1 and C23-4 respectively. The electron transfer in the excitation process could be indicated from hole region $(\mathrm{h}+$, orange) to electron region (e-, green) in the figures. As can be seen from Figure3, the electron excitation in the probe C23 should be charge transfer process.

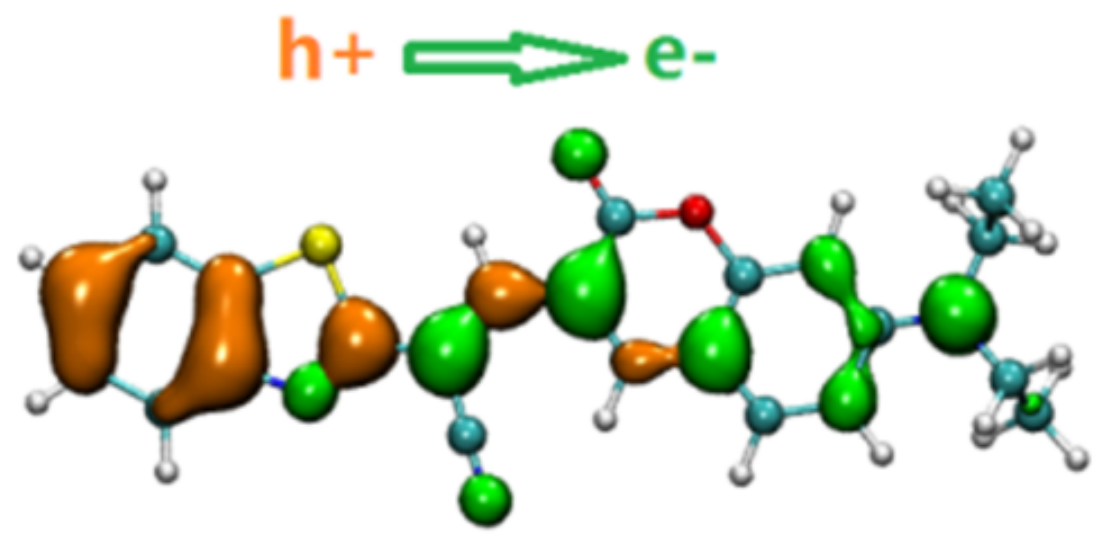




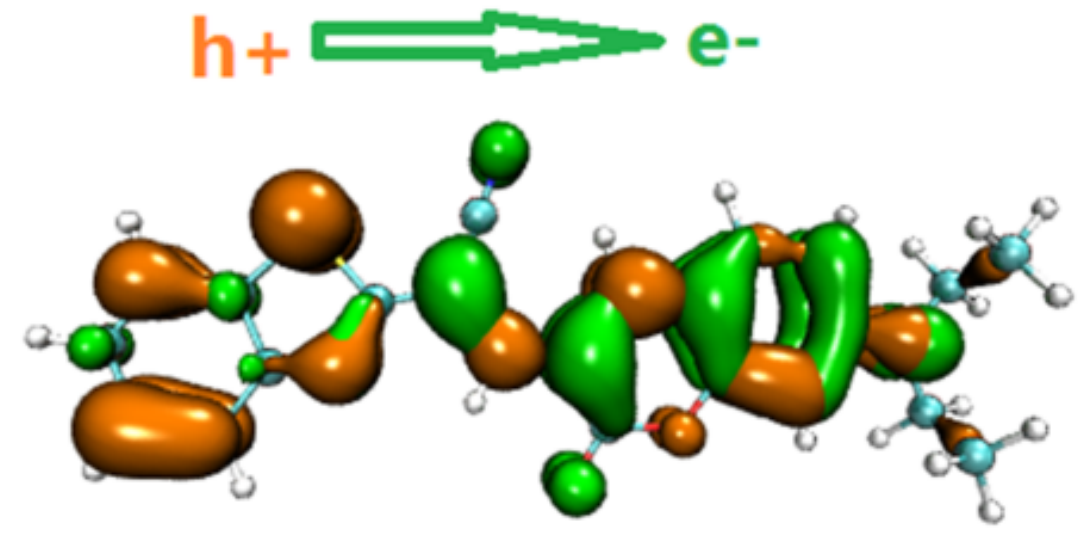

Figure3 Electron density difference between the $\mathrm{S}_{0}$ and $\mathrm{S}_{1}$

(a)C23-1 and (b)C23-4

The probe $\mathrm{C} 23$ could detect $\mathrm{HSA}$ and $\mathrm{SO}_{2}$ simultaneously due to the different fluorescence signal while binding with them. Michael addition reaction between probe $\mathrm{C} 23$ and $\mathrm{SO}_{2}\left(\mathrm{HSO}_{3}{ }^{-1}\right)$ could saturate the rotational bonds in the $\mathrm{C} 23$ and stabilize the conformation which led to the strong fluorescence signal within the blue channel. To understand this mechanism, the conformation and electron excitation process of CS ( $\mathrm{C} 23$ combined with $\mathrm{HSO}_{3}{ }^{-1}$ ) were analyzed using the same methods which were used for $\mathrm{C} 23$ before. The stable conformations of CS structure were reduced to two (CS-1 and CS-2 shown in Figure4). It can be clearly seen that due to the steric hindrance effect of $\mathrm{HSO}_{3}$ group, the rotational $\mathrm{C}-\mathrm{C}$ bonds between the two rings in the probe were saturated and no longer rotated. This Michael addition reaction cut off the non-radiative pathway and led to the blue fluorescence signal within the electron excitation process of CS.

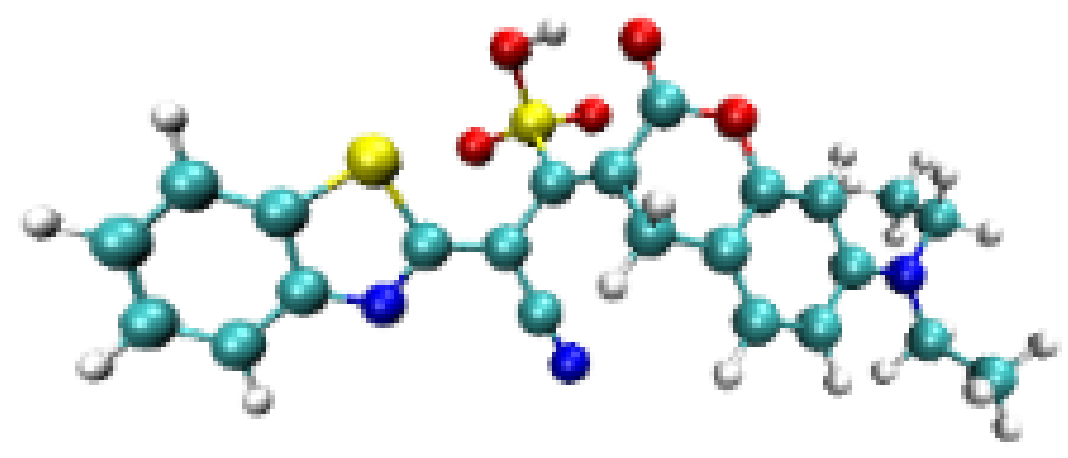




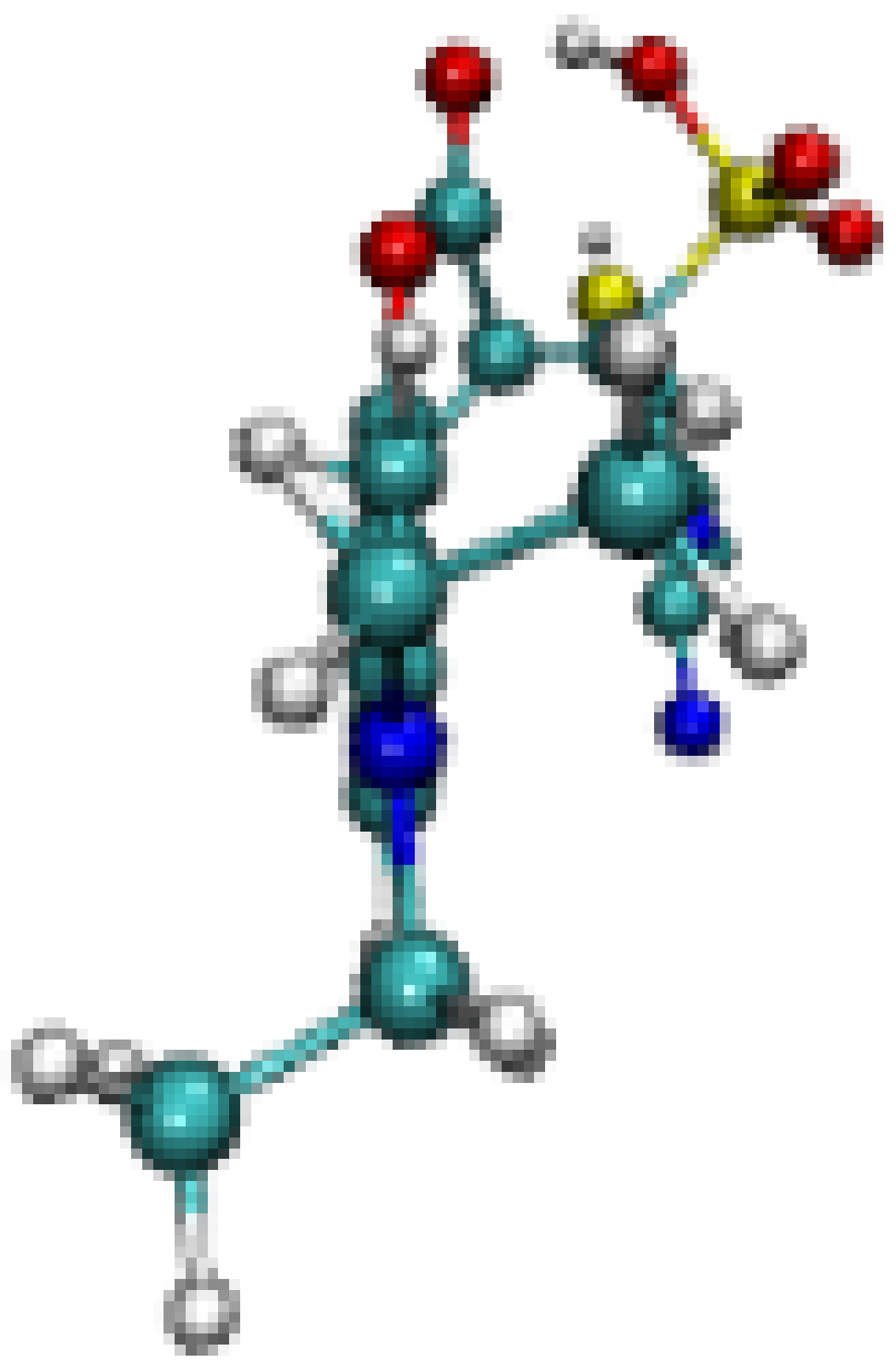




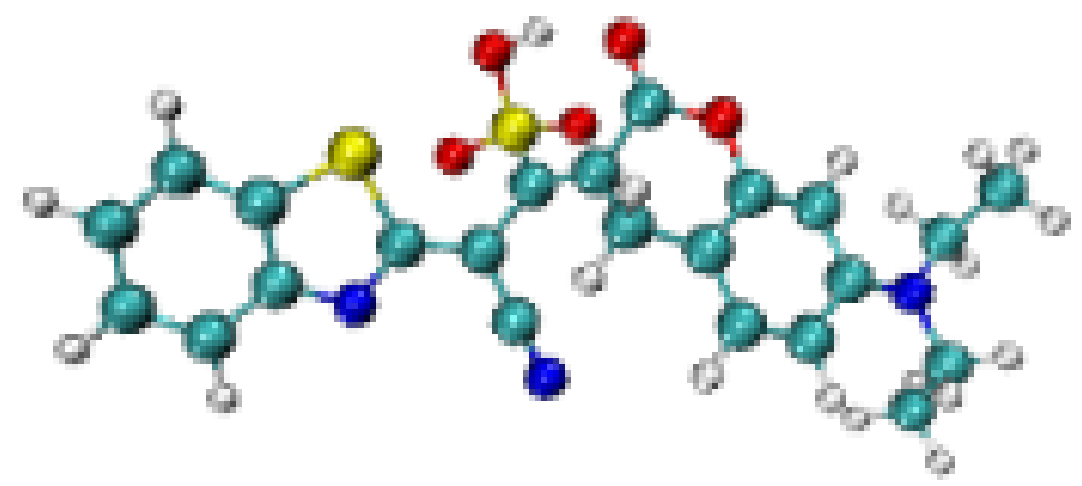




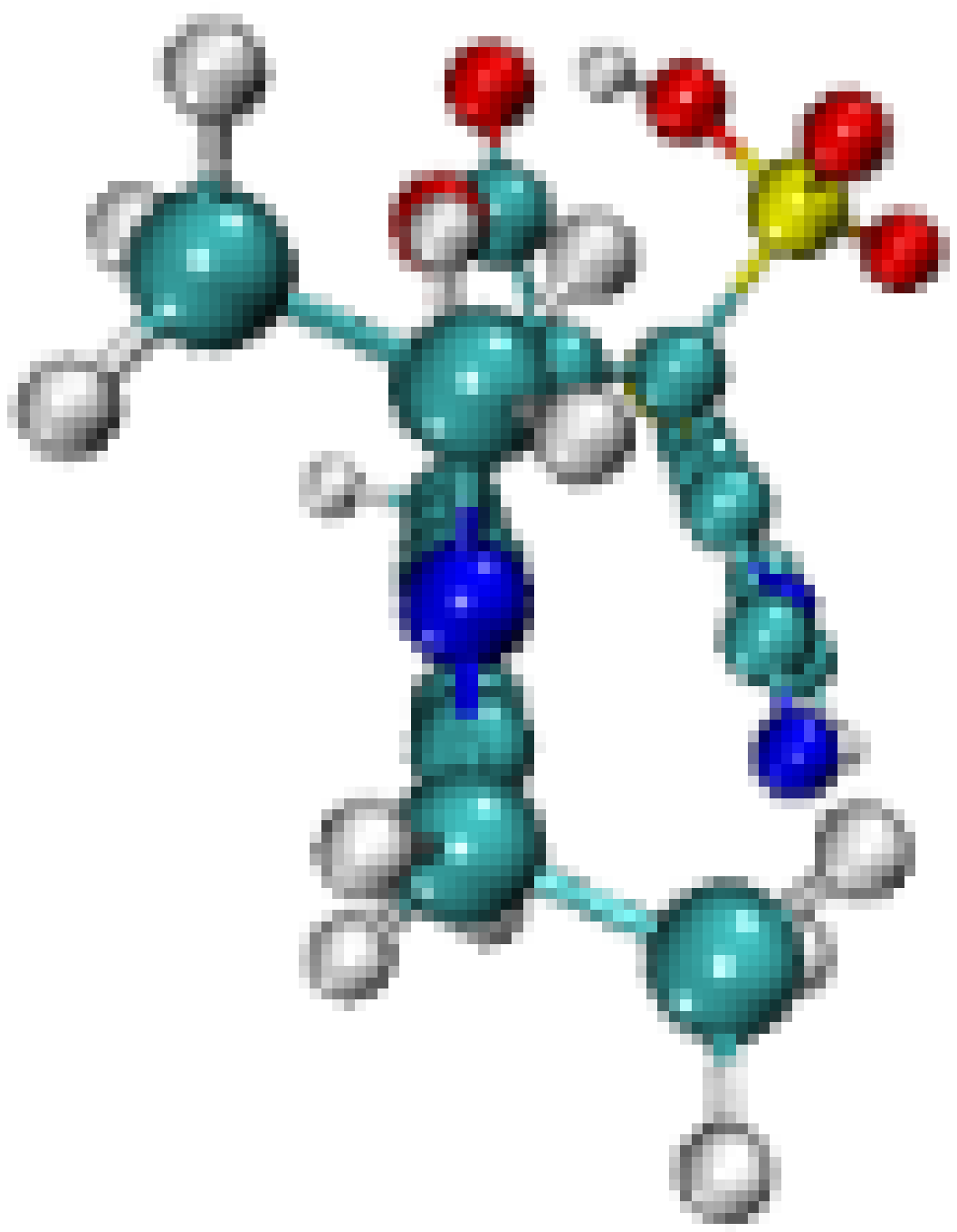

CS-1 CS-2

Figure4 Two stable conformations of CS

The electron density difference between the $\mathrm{S}_{0}$ and $\mathrm{S}_{1}$ of CS- 1 was shown in Figure 5 . The electron excitation in the CS-1 should be taken as charge transfer process but with different direction from C23. 


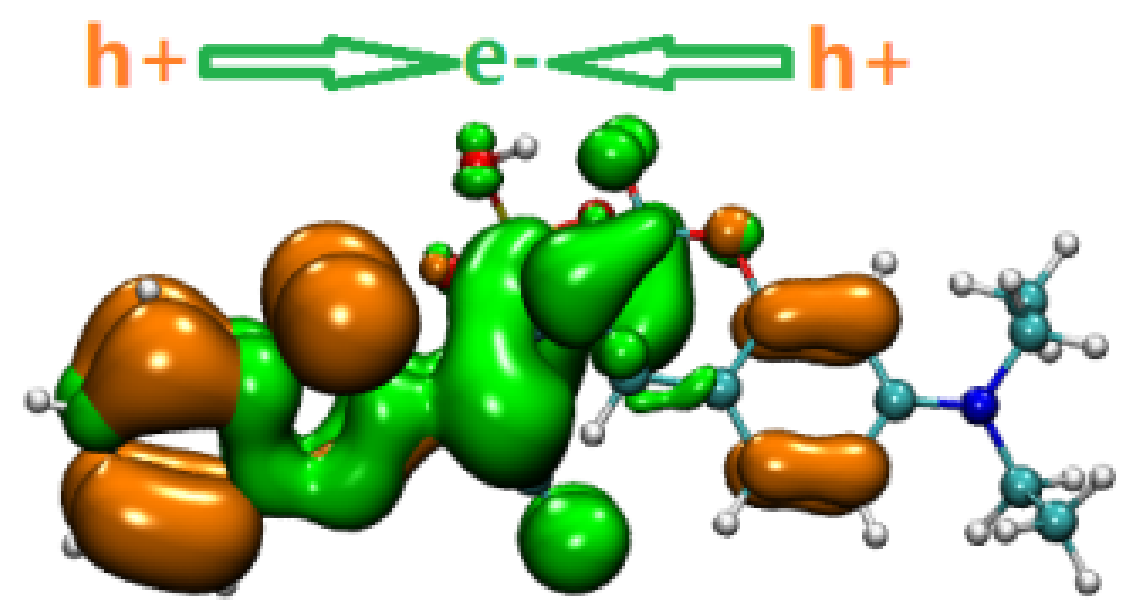

Figure 5 Electron density difference between the $\mathrm{S}_{0}$ and $\mathrm{S}_{1}$ of CS-1

The excitation and emitting fluorescence wavelength within probe C23 and CS were analyzed with ORCA program under wB2GP-PLYP/def2-TZVP and CAM-B3LYP/ def2-TZVP method. The results were summarized in Table 1. The calculated $\lambda_{\text {ex }}$ (wB2GP-PLYP in dmso) values in the table 1 were closed to the experimental values which indicated the red and blue fluorescence channel with probe C23 and CS respectively. Due to unrealized optimization function of first excited state $\mathrm{S}_{1}$ under wB2GP-PLYP function in ORCA program, the CAM-B3LYP functional was utilized for the emitting fluorescence wavelength calculation. Although a clear deviation from the experimental wavelength, a similar trend of wavelength changing with the experimental value was found in the calculation.

Table1 The calculated excitation and emitting fluorescence wavelength (nm) within probe C23 and CS

\begin{tabular}{lllll}
\hline & $\begin{array}{l}\lambda_{\varepsilon \xi}(\omega \mathrm{B} 2 \Gamma \Pi- \\
\Pi \Lambda \Psi \Pi \text { in }\end{array}$ & $\begin{array}{l}\lambda_{\varepsilon \xi}(\omega \mathrm{B} 2 \Gamma \Pi- \\
\Pi \Lambda \Psi \Pi \text { in } \\
\text { gas) }\end{array}$ & $\begin{array}{l}\lambda_{\varepsilon \xi} \text { ("AM- } \\
\text { B3 } \Lambda \Psi \Pi \text { in } \\
\text { gas) }\end{array}$ & $\begin{array}{l}\lambda_{\varepsilon \mu} \text { ("AM- } \\
\text { B3 } \Lambda \Psi \Pi \text { in } \\
\text { gas) }\end{array}$ \\
\hline C23-1 & 731.2 & 552.4 & 981.8 & 1186.6 \\
C23-2 & 733.0 & 562.4 & 988.8 & 1202.7 \\
C23-3 & 737.9 & 575.8 & 994.4 & 1206.2 \\
C23-4 & 738.8 & 585.2 & 1029.9 & 1281.0 \\
CS-1 & 455.9 & 466.1 & 649.2 & 745.2 \\
CS-2 & 454.1 & 465.8 & 645.8 & 743.0 \\
\hline
\end{tabular}

\section{Conclusions}

The different stable conformations of probe $\mathrm{C} 23$ were found through theoretical method which explained the no fluorescent character of the probe itself. The extreme points of electrostatic potential of $\mathrm{C} 23$ showed the potential reaction sites with the $\mathrm{HSA}$ and $\mathrm{SO}_{2}$. After the Michael addition reaction with $\mathrm{SO}_{2}\left(\mathrm{HSO}_{3}{ }^{-1}\right)$, some rotated C-C bonds in $\mathrm{C} 23$ were saturated in CS structure which reduced the number of the stable conformations and led to the blue fluorescence generation. The electron excitation analysis indicated the charge transfer process in the C23 and CS when the molecules were under optical excitation. These results provided the insights in preparing a wide range of fluorescent probes and great potential for widespread industrial applications.

\section{Funding Information}


This work was supported by the Natural Science Foundation of Liaoning Province (JYTQN201923), National Natural Science Foundation of China (81374051)

\section{References}

1 K. Ranguelova, S. Chatterjee, M. Ehrenshaft, D. C. Ramirez, F. A. Summers, M. B. Kadiiska, R. P. Mason, Journal of Biological Chemistry 2010 , 285 , 24195-24205.

2 K. Ranguelova, M. G. Bonini, R. P. Mason, Environmental Health Perspectives 2010 ,118 , 970-975.

3 A. Kumar, M. Triquigneaux, J. Madenspacher, K. Ranguelova, J. J. Bang, M. B. Fessler, R. P. Mason, Redox Biology 2018, $15,327-334$.

4 R. P. Mason, D. Ganini, Free Radical Biology And Medicine 2019 , 131 , 318-331.

5 C. L. Hawkins, M. J. Davies, Journal Of Biological Chemistry 2019, 294 , 19683-19708.

6 J. N. Cobley, H. Husi,Antioxidants 2020,9 ,

7 Z. Liang, Y. Sun, H. Zeng, K. Sun, R. Yang, Z. Li, K. Zhang, X. Chen, L. Qu, Analytical Chemistry2020 , 92, 16130-16137.

8 W. Humphrey, A. Dalke, K. K. Schulten, Journal of Molecular Graphics 1995 , 14, 33 - 38.

9 T. Lu, F. Chen, Journal of Computational Chemistry 2012 , 33 , 580-592.

10 N. M. O'Boyle, T. Vandermeersch, C. J. Flynn, A. R. Maguire, G. R. Hutchison, Journal Of Cheminformatics 2011, 3,

11 C. Bannwarth, S. Ehlert, S. Grimme, Journal Of Chemical Theory And Computation 2019 ,15 , 1652 1671.

12 T. Lu, molclus program, Version 1.9.9.3, http://www.keinsci.com/research/molclus.html (accessed 2021.2.11),

13 F. Neese, Wiley Interdiplinary Reviews Computational Molecular ence 2018 ,8, e1327.

14 S. Grimme, S. Ehrlich, L. Goerigk, Journal of Computational Chemistry 2011, 32 , 1456-1465.

15 F. Weigend, R. Ahlrichs, Physical Chemistry Chemical Physics 2005 , 7 , 3297-3305.

16 L. Goerigk, S. Grimme, Wiley Interdisciplinary Reviews-Computational Molecular Science 2014 , 4 , 576-600.

17 M. Casanova-Paez, L. Goerigk, Journal Of Chemical Physics 2020 , 153 , 